Ann. Génét. Sél. anim., I97I, 3 (4), 521-525.

\title{
DEUX GAS NOUVEAUX DE FUSION CENTRIQUE CHEZ LES BOVINS
}

\author{
C.-P. POPESCU \\ avec la collaboration technique de $\mathrm{M}^{\mathrm{me}}$ Jeannine Boscher \\ Union nationale des Coopératives d'Élevage et Insémination artificielle et \\ Laboratoire de Génétique factorielle, \\ Centre national de Recherches zootechniques, I. N. R. A., \\ 78 - Jouy-en-Josas
}

\section{RÉSUMÉ}

Deux cas de fusion centrique sont décrits dans la race montbéliarde. Selon l'idiogramme établi, la fusion centrique implique, comme dans les autres races où elle a été décrite, les chromosomes I et 29 .

La translocation de type robertsonien entre 2 chromosomes acrocentriques avec la formation d'un chromosome méta- ou submétacentrique est aussi appelée fusion centrique. Sur le nombre réduit d'anomalies de structure décrites chez les bovins elle est actuellement la plus fréquente.

Gustavsson et RocквоRn (I964) sont les premiers qui la décrivent chez trois animaux de la race rouge suédoise. Il s'agissait d'une fusion centrique avec la formation d'un nouveau submétacentrique et la réduction du nombre de base à 59, à l'état hétérozygote. Une vaste étude entreprise par la suite, sur un grand nombre d'animaux, a révélé que, dans cette race, I 3 p. Ioo des animaux portent la fusion centrique à l'état hétérozygote et 0,5 p. soo à l'état homozygote (GusTAVsson, I966, I969).

La même anomalie est trouvée par Herschler et Fechheimer (i966) chez une femelle free-martin, issue d'une naissance triple. RUGIATI et FEDRIGo (I967) observent une fusion centrique à l'état homozygote chez un taureau romagnol, achondroplasique.

RIEck et al. (1968) la trouvent chez une femelle pie rouge allemande et chez 3 de ses descendants. Amrud (1969) étudie 430 animaux de race rouge norvégienne et trouve 4,3 p. Ioo d'animaux hétérozygotes pour la fusion centrique mais aucun homozygote, ce qui indique une fréquence moins importante que dans la race rouge suédoise. Récemment, HARVEY (I97I) décrit une fusion centrique chez un taureau charolais et 8 de ses descendants. 
A l'exception de l'animal achondroplasique décrit par Rugiati et Fedrigo (1967) et des trois premiers étudiés par Gustavsson et RockBorn (I964) qui étaient atteints de leucémie lymphatique, tous les autres animaux trouvés porteurs de la fusion centrique étaient phénotypiquement normaux. Mais, étudiant les effets de cette anomalie, Gustavsson (197I) trouve un taux d'élimination plus grand ainsi qu'une fertilité basse, chez les filles des taureaux affectés. En raison de ces effets et de la fréquence relativement grande dans certaines races bovines, l'étude de cette anomalie s'avère importante.

Dans la présente note sont décrits deux animaux porteurs d'une fusion centrique, appartenant à la race montbéliarde.

\section{MATÉRIEL ETT MÉTHODE}

L'étude porte sur deux taureaux montbéliards âgés respectivement de 3 et II ans, phénotypiquement normaux et de fertilité normale. Des cultures de sang ont été faites selon la méthode de Moorhpad et al. (1960), modifiée par Nichois et al. (I962). Environ 30 métaphases ont été photographiées au grossissement I $25^{\circ}$ x sur une pellicule Kodak Microfile. Un idiogramme a été établi, basé sur une méthode décrite antérieurement (Popescu, I969). La longueur moyenne de chaque chromosome est exprimée en valeur relative, comme part pour mille de la longueur totale du complément haploïde.

La variance et l'erreur standard de la moyenne ont été calculées pour chaque chromosome.

\section{RÉSULTATS ET DISCUSSION}

Dans toutes les cellules étudiées, chez les deux animaux, le nombre chromosomique est de 59 parmi lesquels 47 autosomes et 2 chromosomes sexuels (fig. I $a$ et $b$ ). La réduction du nombre chromosomique, normalement de 60 pour cette espèce, est due à une fusion centrique survenue entre 2 autosomes qui ont formé un chromosome nouveau submétacentrique.

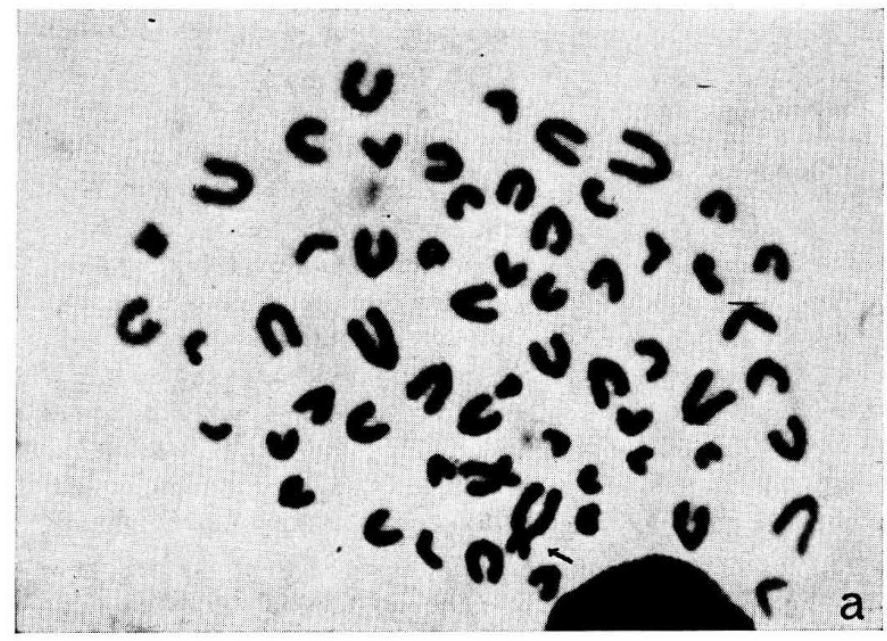

FIG. I

a) Métaphase portant la fusion centrique $(2 n=59)$

Le chromosome fusionné est marqué par une fièche 


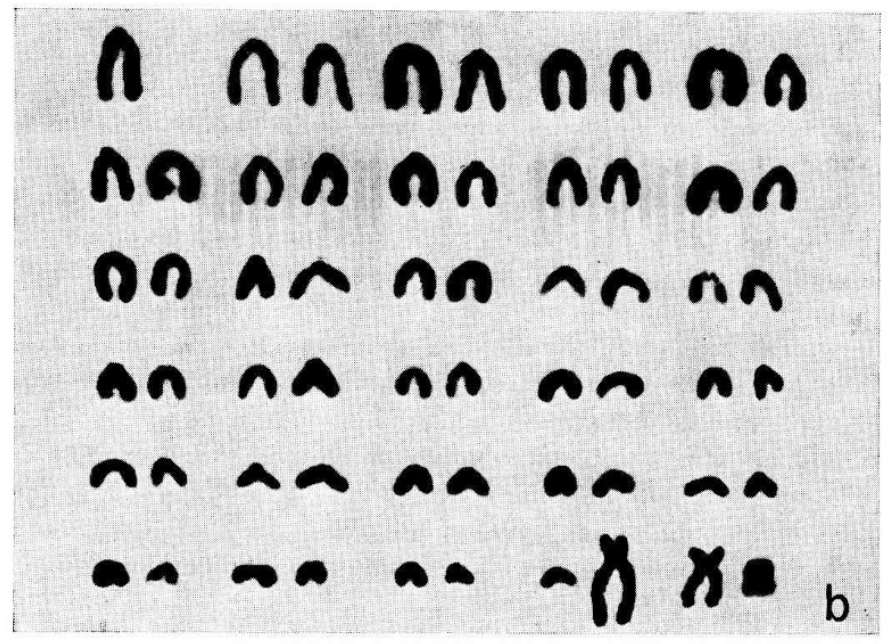

FIG. I

b) Caryotype d'une cellule avec la fusion centrique

TABLEAU I

Valeurs relatives moyennes des chromosomes du complément haploïde établies sur 5 cellules

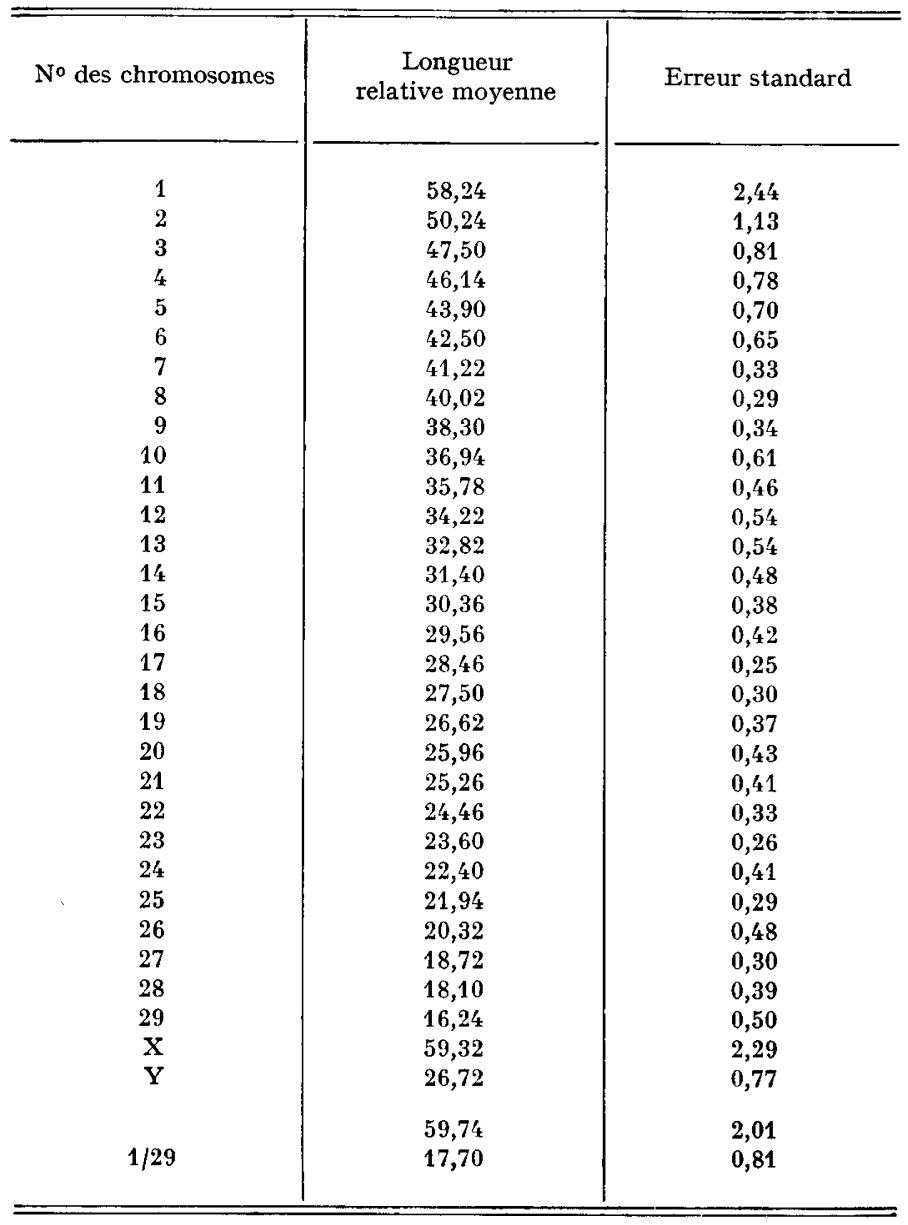


Les valeurs moyennes des longueurs relatives des deux bras du chromosome fusionné, présentées dans l'idiogramme (tabl. I), correspondent au plus grand et, respectivement, au plus petit des chromosomes du complément haploïde. Les 5 cellules mesurées dans cet idiogramme appartenaient à l'animal âgé de 3 ans. Des mesures pratiquées sur des cellules de l'autre animal qui ne figurent pas dans ce tableau, ont donné des chiffres semblables.

La participation du chromosome $\mathrm{n}^{\circ} \mathrm{I}$, le plus grand du complément, dans cette fusion, est sans ambiguité, puisque sa taille est nettement supérieure à celle de la deuxième paire et sa valeur relative assez proche de la valeur du bras long du chromosome fusionné. Quant au petit bras du chromosome fusionné, bien qu'il y ait une certaine différence par excès entre sa valeur relative et celle du plus petit chromosome due aux erreurs de mesure, son identité avec ce chromosome paraît la plus vraisemblable.

En effet, dans son ensemble le chromosome fusionné paraît surévalué quant à sa longueur relative puisque même son bras long est plus grand que le chromosome $\mathrm{I}$, alors qu'une fusion centrique implique toujours la perte d'un petit fragment centromérique. De plus, il est possible qu'en mesurant le chromosome fusionné, on ait favorisé le petit bras, en raison de la difficulté de préciser la position exacte du centromère.

Se basant sur un idiogramme établi pour des cellules normales et des cellules à 59 chromosomes, Gustavsson (r 969 ) trouve que la fusion centrique présente dans la race rouge suédoise, implique le plus grand et le plus petit chromosome du complément (I/29). AMRUD (I969) affirme également qu'il s'agit du même type de fusion dans la race rouge norvégienne et qu'elle aurait été introduite par des taureaux de race rouge suédoise, qui avaient contribué à la formation de cette race.

Sans avoir fait de mesures, Rugiati et Fedrigo (1967) pensent, de leur côté, que la fusion centrique observée dans la race romagnole se serait produite entre les chromosomes $\mathrm{n}^{0}$ I et 28 . Dans les autres cas décrits dans la race pie rouge allemande (RIECK et al. I968) et charolaise (HARVEY, I97I) la fusion centrique implique toujours les chromosomes I et 29.

L'hypothèse d'une anomalie chromosomique du même type, impliquant les mêmes chromosomes, apparue par hasard dans des races et des pays différents, est peu probable. On peut plutôt supposer que cette anomalie remonte plus loin dans la phylogenèse de l'espèce, chez un ancêtre existant avant la formation des races actuelles.

Reçu pour publication en novembre 1971.

\section{SUMMARY}

\section{TWO NEW CASES OF CENTRIC FUSION IN CATTLE}

Two new cases of centric fusion are described in montbéliard breed of cattle.

Idiogram shows that chromosomes I and 29 are involved in the centric fusion, as in other cattle breeds where it has soon been found.

\section{RÉFÉRENCES BIBLIOGRAPHIQES}

Amrud J., 1969. Centric fusion of chromosomes in norvegian red cattle (N. R. C.). Hereditas 62 (3), 293-302

Gustavsson I., Rockborn G., I964. Chromosome abnormality in three cases of lymphatic leukaemia in cattle. Nature (Lond.), 203, 990 .

Gustavsson I., 1966. Chromosome abnormality in cattle. Nature (Lond.), 211, 865-866. 
Gustavsson I., I969. Cytogenetics, distribution and phenotypic effects of a translocation in swedish cattle. Hereditas, 63, 68-169.

Gustavsson I., 197I. Culling rates in daughters of sires with a translocation of centric fusion type. Hereditas, 67, 65-74.

HARVEY M. J. O., I97I. An autosomal translocation in Charolais breed of cattle. Vet. Rec., 89, IIO-III. Herschler M. S., Fechheimer N. S., ig66. Centric fusion of chromosomes in a set of bovine triplets. Cytogenetics, 5, 307-312.

Moorhead P. S., Nowell P. C., Mellman W. J., Battips D. M., Hungerford D. A., Ig6o. Chromosome preparation of leukocytes cultured in human peripheral blood. Exp. Cell. Res., 20, 6r3-6r6.

Nichols W. W., Levan A., Lawrence W. C., ig62. Bovine chromosomes by the peripheral blood method. Hereditas, 48, 536-538.

Popescu C. P., r969. Idiograms of Yak (Bos grunniens) cattle (Bos taurus) and their hybrid. Ann. Génét. Sél. anim., 1, 207-2I7.

Rieck G. W., Höhn H., Herzog A., I968. Familial occurrence of centromeric chromosome fusion in cattle. Zuchthygiene, 3, II7-I82.

Rugiati S., Fedrigo M., I967. Alterazione cromosomica riscontrata in un toro acondroplasico di razza Romagnola. L'Ateneo Parmense. Acta Bio, Medica, 38, r-7. 